FNT/T 2003/05

\title{
Higher-order QED corrections to $W$-boson mass determination at hadron colliders
}

\author{
C.M. Carloni Calame, ${ }^{1,2}$ G. Montagna, ${ }^{2,1}$ O. Nicrosini, ${ }^{1,2}$ and M. Treccani ${ }^{2}$ \\ ${ }^{1}$ Istituto Nazionale di Fisica Nucleare, \\ Sezione di Pavia, via A. Bassi 6, I-27100, Pavia, Italy \\ ${ }^{2}$ Dipartimento di Fisica Nucleare e Teorica, \\ Università di Pavia, via A. Bassi 6, I-27100, Pavia, Italy
}

(Dated: November 15, 2018)

\begin{abstract}
The impact of higher-order final-state photonic corrections on the precise determination of the $W$-boson mass at the Tevatron and LHC colliders is evaluated. In the presence of realistic selection criteria, the shift in the $W$ mass from a fit to the transverse mass distribution is found to be about $10 \mathrm{MeV}$ in the $W \rightarrow \mu \nu$ channel and almost negligible in the $W \rightarrow e \nu$ channel. The calculation, which is implemented in a Monte Carlo event generator for data analysis, can contribute to reduce the uncertainty associated to the $W$ mass measurement at future hadron collider experiments.
\end{abstract}

PACS numbers: 12.15.Lk,13.40.Ks,14.70.Fm

Keywords: hadron collision, $W$ boson, QED corrections, Monte Carlo 
Precision tests of the Standard Model require a more and more accurate knowledge of the basic parameters of the theory. In particular, future measurements of the $W$-boson and top quark masses at the Tevatron and the LHC colliders are expected to considerably improve the present indirect bound on the Higgs-boson mass from electroweak precision data. As recently discussed in the literature [1], a precision of $27 \mathrm{MeV}(16 \mathrm{MeV})$ for the $W$ mass $M_{W}$ is the target value for Run IIa (Run IIb) of the Tevatron. An accuracy of $15 \mathrm{MeV}$ is the final goal of LHC [2].

In order to measure $M_{W}$ with such a high precision in a hadron collider environment, it is mandatory to keep under control higher-order QCD and electroweak radiative corrections to the $W$ and $Z$ production processes. The status of QCD corrections to weak boson production in hadronic collisions is reviewed in Ref. [3], while recent progress in the calculation of electroweak corrections, as achieved by means of independent calculations [4, 5, 6], is summarized in Ref. 7]. As shown in Refs. [4, 5, 6, 7], electroweak corrections are dominated by photon radiation effects and, in particular, by final-state photon emission, which gives rise to collinear logarithms of the form $\alpha / \pi \log \left(\hat{s} / m_{l}^{2}\right)$, where $\hat{s}$ is the effective centre of mass (c.m.) energy and $m_{l}$ is the mass of the final-state lepton. This poses the question of the impact of higher-order (i.e. beyond order $\alpha$ ) leading logarithmic corrections due to multi-photon radiation. A first attempt toward the inclusion of $\mathcal{O}\left(\alpha^{2}\right)$ QED corrections was the calculation of the double-bremsstrahlung matrix elements $q \overline{q^{\prime}} \rightarrow W \rightarrow l \nu \gamma \gamma$ and $q \bar{q} \rightarrow \gamma, Z \rightarrow l^{+} l^{-} \gamma \gamma(l=e, \mu)$ performed in Ref. [8]. The aim of the present work is to evaluate the impact of higher-order final-state QED corrections on the $W$ mass determination at hadron colliders, by including both real bremsstrahlung and virtual corrections. To this end, a Parton Shower (PS) approach in QED [9] is employed to simulate multi-photon radiation effects. An independent calculation of multi-photon radiative corrections in leptonic $W$ decays has appeared very recently [10], but without quantifying their impact on the $W$ mass measurement. The uncertainty in the $W$ mass due to higher-order QED effects is presently estimated by the CDF collaboration at the Tevatron to be $20 \mathrm{MeV}$ in the $W \rightarrow e \nu$ channel, and $10 \mathrm{MeV}$ in the $W \rightarrow \mu \nu$ channel [11]. An uncertainty of $12 \mathrm{MeV}$ is assigned

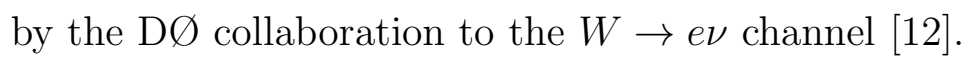

From our analysis, we find that the shift in the fitted $W$ mass is about $10 \mathrm{MeV}$ in the $W \rightarrow \mu \nu$ channel and almost negligible in the $W \rightarrow e \nu$ channel. The inclusion of the present calculation in the experimental analysis would reduce significantly such a theoretical 
uncertainty in future improved measurements of the $W$ mass at hadron colliders.

An appropriate theoretical tool to compute photonic radiative corrections in the leading log approximation is the QED PS approach [9]. It consists in a numerical solution of the QED Gribov-Lipatov-Altarelli-Parisi evolution equation for the charged lepton Structure Function $D\left(x, Q^{2}\right)$ in the non-singlet channel. The solution can be cast in the form [9]

$$
\begin{aligned}
& D\left(x, Q^{2}\right)=\Pi\left(Q^{2}, m^{2}\right) \delta(1-x) \\
+ & \left(\frac{\alpha}{2 \pi}\right) \int_{m^{2}}^{Q^{2}} \Pi\left(s, s^{\prime}\right) \frac{d s^{\prime}}{s^{\prime}} \Pi\left(s^{\prime}, m^{2}\right) \int_{0}^{x_{+}} d y P(y) \delta(x-y) \\
+ & \left(\frac{\alpha}{2 \pi}\right)^{2} \int_{m^{2}}^{Q^{2}} \Pi\left(s, s^{\prime}\right) \frac{d s^{\prime}}{s^{\prime}} \int_{m^{2}}^{s^{\prime}} \Pi\left(s^{\prime}, s^{\prime \prime}\right) \frac{d s^{\prime \prime}}{s^{\prime \prime}} \Pi\left(s^{\prime \prime}, m^{2}\right) \\
& \int_{0}^{x_{+}} d x_{1} \int_{0}^{x_{+}} d x_{2} P\left(x_{1}\right) P\left(x_{2}\right) \delta\left(x-x_{1} x_{2}\right)+\cdots
\end{aligned}
$$

where $\Pi\left(s_{1}, s_{2}\right)=\exp \left[-\frac{\alpha}{2 \pi} \int_{s_{2}}^{s_{1}} \frac{d s^{\prime}}{s^{\prime}} \int_{0}^{x_{+}} d z P(z)\right]$ is the Sudakov form factor, $P(z)$ is the $e \rightarrow e+\gamma$ splitting function and $x_{+}$is an infrared regulator, separating the soft+virtual region from hard bremsstrahlung. Equation (11) allows to compute $D\left(x, Q^{2}\right)$ by means of a Monte Carlo algorithm which, as shown in Ref. [9], simulates the emission of a shower of (real and virtual) photons by a charged fermion and accounts for exponentiation of soft photons and re-summation of collinear logarithms due to multiple hard bremsstrahlung. A remarkable advantage of the PS algorithm with respect to a strictly collinear approximation is the possibility of generating transverse momentum $p_{T}$ of fermions and photons at each branching, thus allowing an exclusive event generation suitable to implement experimental cuts according to a realistic event selection. The generation of transverse degrees of freedom can be performed according to different recipes, as described in detail in Ref. [9]. Here, we generate photon angular variables according to the leading pole behavior $1 /\left(1-\beta_{l} \cos \vartheta_{l \gamma}\right)$, where $\beta_{l}$ is the lepton velocity and $\vartheta_{l \gamma}$ is the relative lepton-photon angle.

A simple recipe to evaluate final-state corrections to $p \stackrel{(-)}{p} \rightarrow W \rightarrow \nu l$ consists in attaching a single structure function $D\left(x, Q^{2}\right)$ to the lepton coming from the $W$ decay. Needless to say, this amounts to neglect photonic corrections due to initial-state radiation, initial-final-state interference and $W$-boson emission. However, it is known that radiation from an internal off-shell particle can not contribute to leading logarithmic corrections, which are the main concern of the present study. Further, initial-state photon radiation requires an appropriate treatment, since radiation off quarks gives rise to quark mass singularities which, as discussed in Refs. [4, 5, 6, 7], can be reabsorbed in Parton Distribution Functions (PDF), in analogy 
TABLE I: Comparison between the present calculation (HORACE) and WGRAD [5, 14] for the $p \stackrel{(-)}{p} \rightarrow W \rightarrow l \nu, l=e, \mu$ cross sections (in pb), at the Tevatron Run II $(\sqrt{s}=2 \mathrm{TeV})$ and the LHC $(\sqrt{s}=14 \mathrm{TeV})$.

\begin{tabular}{lcccc}
\hline \hline & \multicolumn{2}{c}{$\sqrt{s}=2 \mathrm{TeV}$} & \multicolumn{2}{c}{$\sqrt{s}=14 \mathrm{TeV}$} \\
\cline { 2 - 5 } & $e$ & $\mu$ & $e$ & $\mu$ \\
\hline WGRAD Born & \multicolumn{2}{c}{$441.7(1)$} & \multicolumn{2}{c}{$1906(1)$} \\
WGRAD & $418.3(4)$ & $429.4(3)$ & $1800(2)$ & $1845(2)$ \\
WGRAD final-state & $419.7(1)$ & $430.0(1)$ & $1808(1)$ & $1854(1)$ \\
HORACE Born & & $441.6(1)$ & & $1905(1)$ \\
HORACE $\mathcal{O}(\alpha)$ & $419.4(1)$ & $429.9(1)$ & $1806(1)$ & $1853(1)$ \\
HORACE exponentiated & $419.5(1)$ & $430.0(1)$ & $1808(1)$ & $1853(1)$ \\
\hline \hline
\end{tabular}

to gluon emission in QCD. These considerations imply that the treatment of final-state photon radiation alone is not gauge invariant. Nevertheless, it can be easily checked, by comparing the PS spectrum with the gauge-invariant factor for collinear photon emission by a fermion [13], that gauge violations are confined to the next-to-leading order corrections, which are beyond the approximation of the present analysis. This issue is further discussed in the following, by means of a quantitative comparison with the independent, gauge-invariant calculation of Ref. [5].

In order to quantify the effect of higher-order final-state QED corrections on the $W$ mass determination, we developed a Monte Carlo event generator following the approach described above and performed a number of Monte Carlo experiments. The technical details of the Monte Carlo code HORACE (Higher Order RAdiative CorrEctions) will be presented elsewhere. Before the phenomenological analysis, we performed a tuned comparison between the predictions of HORACE and those of WGRAD [5, 14], to verify the accuracy of our calculation. The results of such a comparison are shown in Tab. 【 using default PDFs, input parameters and cuts as in Ref. [14]. WGRAD includes the full set of $\mathcal{O}(\alpha)$ electroweak radiative corrections to $W$ production (second line in Tab. I) but it also gives the possibility to select the effect of a gauge-invariant subset due to final-state corrections (WGRAD finalstate in Tab. (1). Therefore, the difference, at a few per mille level, between WGRAD and WGRAD final-state points out, when comparing with the Born predictions, the dominance 
of final-state radiation within the full set of $\mathcal{O}(\alpha)$ electroweak corrections. On the other hand, it can be seen that the predictions by WGRAD final-state are in very good agreement with our results by HORACE $\mathcal{O}(\alpha)$, which is an order $\alpha$ expansion of the complete PS algorithm. Since the differences are well below the $0.1 \%$ level, this comparison demonstrates that the gauge-invariance violations present in our approach are numerically negligible. The contribution of higher-order effects can be seen by comparing HORACE $\mathcal{O}(\alpha)$ with our complete predictions given by HORACE exponentiated. Their effect on the integrated cross section is tiny, within $0.1 \%$. For the sake of completeness, we performed also comparisons between WGRAD and HORACE at the level of differential distributions, such as lepton transverse momentum and transverse mass distributions, finding perfect agreement.

Having established the physical and technical accuracy of our calculation, we move to the analysis of the $W$ mass shift due to higher-order corrections. The input parameters used in the simulations are:

$$
\begin{aligned}
& m_{\nu_{l}}=0 \quad m_{e}=0.511 \times 10^{-3} \mathrm{GeV} \\
& m_{\mu}=0.10565836 \mathrm{GeV} \\
& \alpha^{-1}=137.03599976 \quad G_{\mu}=1.16639 \times 10^{-5} \mathrm{GeV}^{-2} \\
& \alpha_{s}=0.1185 \\
& M_{W}=80.423 \mathrm{GeV} \quad M_{Z}=91.1882 \mathrm{GeV} \\
& \sin ^{2} \theta_{W}=1-\frac{M_{W}^{2}}{M_{Z}^{2}} \quad \Gamma_{W}=\frac{3 G_{\mu} M_{W}^{3}}{2 \sqrt{2} \pi}\left(1+\frac{2 \alpha_{s}}{3 \pi}\right)
\end{aligned}
$$

We adopt the $G_{\mu}$ scheme and fixed-width scheme in our calculation. At the parton level, we consider the processes

$$
\begin{gathered}
u+\bar{d} \rightarrow W^{+} \rightarrow l^{+}+\nu_{l} \quad u+\bar{s} \rightarrow W^{+} \rightarrow l^{+}+\nu_{l} \\
c+\bar{d} \rightarrow W^{+} \rightarrow l^{+}+\nu_{l} \quad c+\bar{s} \rightarrow W^{+} \rightarrow l^{+}+\nu_{l}
\end{gathered}
$$

and their charge conjugate, with $l=e, \mu$ and CKM matrix elements according to Ref. [15]. The results for the processes $p \bar{p} \rightarrow W \rightarrow l+\nu$ (Tevatron) and $p p \rightarrow W \rightarrow l+\nu$ (LHC) are obtained by convoluting the parton-level matrix element with CTEQ6 PDFs [16]. The virtuality scale $Q^{2}$ is set to be $Q^{2}=\hat{s}, \hat{s}$ being the effective c.m. energy after gluon radiation, in both PDFs and lepton Structure Function. The c.m. energies considered are $\sqrt{s}=2 \mathrm{TeV}$ for the Tevatron and $\sqrt{s}=14 \mathrm{TeV}$ for the LHC. 
To model the acceptance cuts used by the $\mathrm{CDF}$ and $\mathrm{D} \varnothing$ collaborations in their $W$ mass analyses, we impose the following transverse momentum $\left(p_{T}\right)$ and pseudo-rapidity $(\eta)$ cuts:

$$
p_{T}(l)>25 \mathrm{GeV} \quad|\eta(l)|<1.2 \quad \not p_{T}>25 \mathrm{GeV}
$$

However, in order to perform a realistic phenomenological analysis and study the dependence of the $W$ mass shift from detector effects, we implement, in addition to the above cuts, the lepton identification requirements quoted in Table I of Ref. [5]. According to these criteria, electron and photon four-momenta are recombined for small opening angles between the two particles, consistently with a calorimetric particle identification, while muons are identified as hits in the muon chambers with an associated track consistent with a minimum ionizing particle. Furthermore, we simulate uncertainties in the energy and momentum measurements of the charged leptons in the detector by means of a Gaussian smearing of the particle four-momenta, using as standard deviation values the specifications relative to electrons and muons for the Run II DØ detector [17].

The strategy followed by the $\mathrm{CDF}$ and $\mathrm{D} \varnothing$ collaborations to extract $M_{W}$ from the data is to perform a maximum likelihood fit to the transverse mass distribution of the final-state lepton pair or to the transverse momentum of the charged lepton. Here we consider the transverse mass, which is the preferred quantity to determine the $W$ mass and is defined as

$$
M_{T}=\sqrt{2 p_{T}(l) p_{T}(\nu)\left(1-\cos \phi^{l \nu}\right)}
$$

where $p_{T}(l)$ and $p_{T}(\nu)$ are the transverse momentum of the lepton and neutrino, and $\phi^{l \nu}$ is the angle between the lepton and the neutrino in the transverse plane. The transverse mass distribution, as obtained by our simulation, is shown in Fig. 固at $\sqrt{s}=2 \mathrm{TeV}$. The distribution without lepton identification requirements and smearing effects (solid histogram) is compared to the distribution including lepton identification criteria (markers) and detector resolutions (shaded histogram). The shape of the $M_{T}$ spectrum is considerably modified by detector resolution effects, in agreement with the results shown in Refs. [11, 12]. The arrows in Fig. 1 1 select the range $65 \mathrm{GeV}<M_{T}<100 \mathrm{GeV}$, which is used by CDF collaboration in its $W$ mass analysis and we also adopt in the fitting procedure described below.

To evaluate the shift induced by higher-order corrections on the $W$ mass, we perform binned $\chi^{2}$ fits and binned maximum likelihood fits to the $M_{T}$ distribution, in complete analogy with the experimental fitting procedure. Here we show only the results of the $\chi^{2}$ 


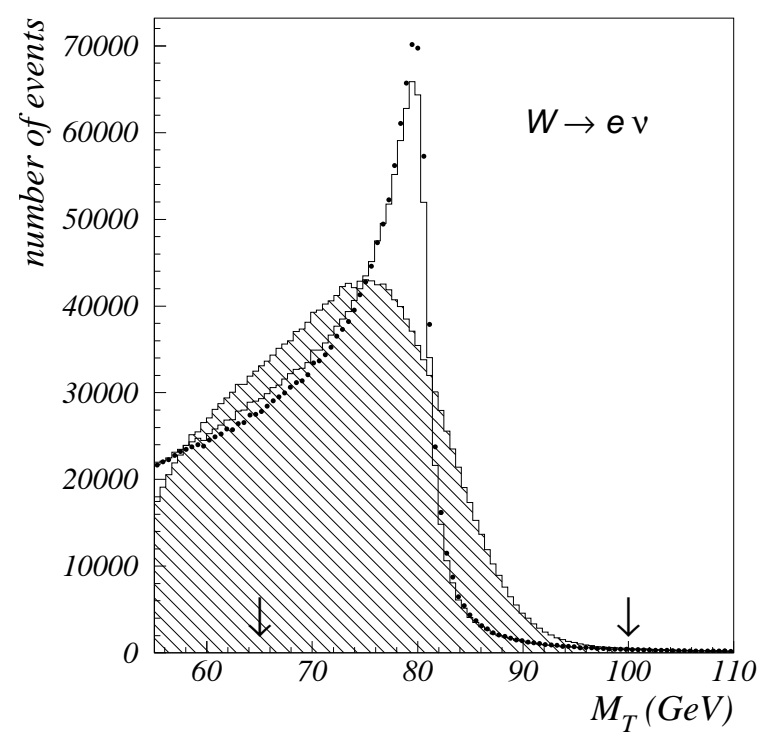

FIG. 1: The transverse mass distribution without lepton identification criteria and detector resolutions (solid histogram), with lepton identification criteria (markers) and with detector resolutions (shaded histogram), in the $W \rightarrow e \nu$ channel at $\sqrt{s}=2 \mathrm{TeV}$. Arrows indicate the considered fit region.

fits, because the results of the maximum likelihood fits are in perfect agreement with the former. Using HORACE, we generate a sample of pseudo-data and calculate with high numerical precision the $m_{T}$ spectrum (binned into 100 bins) at the Born level and for a fixed, "physical" value of the $W$ mass, i.e. $M_{W}^{\text {ref }}=80.423 \mathrm{GeV}$. Next, we compute the $m_{T}$ spectrum including $\mathcal{O}(\alpha)$ leading-log corrections for 20 hypothesized $W$ mass values, with a spacing of $5 \mathrm{MeV}$ for the $W \rightarrow e \nu$ channel and $10 \mathrm{MeV}$ for the $W \rightarrow \mu \nu$ channel. We then normalize the spectra within the fit interval and we calculate, for each $M_{W}$ value, the $\chi^{2}$ as

$$
\chi^{2}=\sum_{i}\left(\sigma_{i, \alpha}-\sigma_{i, B o r n}\right)^{2} /\left(\Delta \sigma_{i, \alpha}^{2}+\Delta \sigma_{i, B o r n}^{2}\right)
$$

where $\sigma_{i, B o r n}$ and $\sigma_{i, \alpha}$ are the Monte Carlo predictions for the $i^{\text {th }}$ bin at the Born and $\mathcal{O}(\alpha)$ level, respectively, and $\Delta \sigma_{i, B o r n}, \Delta \sigma_{i, \alpha}$ the corresponding statistical errors due to numerical integration. This allows to quantify the mass shift due to $\mathcal{O}(\alpha)$ corrections. The shift due to higher-order corrections is derived according to the same procedure, by generating a sample of pseudo-data for the $M_{T}$ distribution at $\mathcal{O}(\alpha)$ and fitting them in terms of the $M_{T}$ spectrum obtained including higher-order corrections for 10 hypothesized $W$ mass values. In this case, we use $1 \mathrm{MeV}$ spacing between masses. Figure 2] shows the $\Delta \chi^{2}=\chi^{2}-\chi_{\min }^{2}$ distributions 

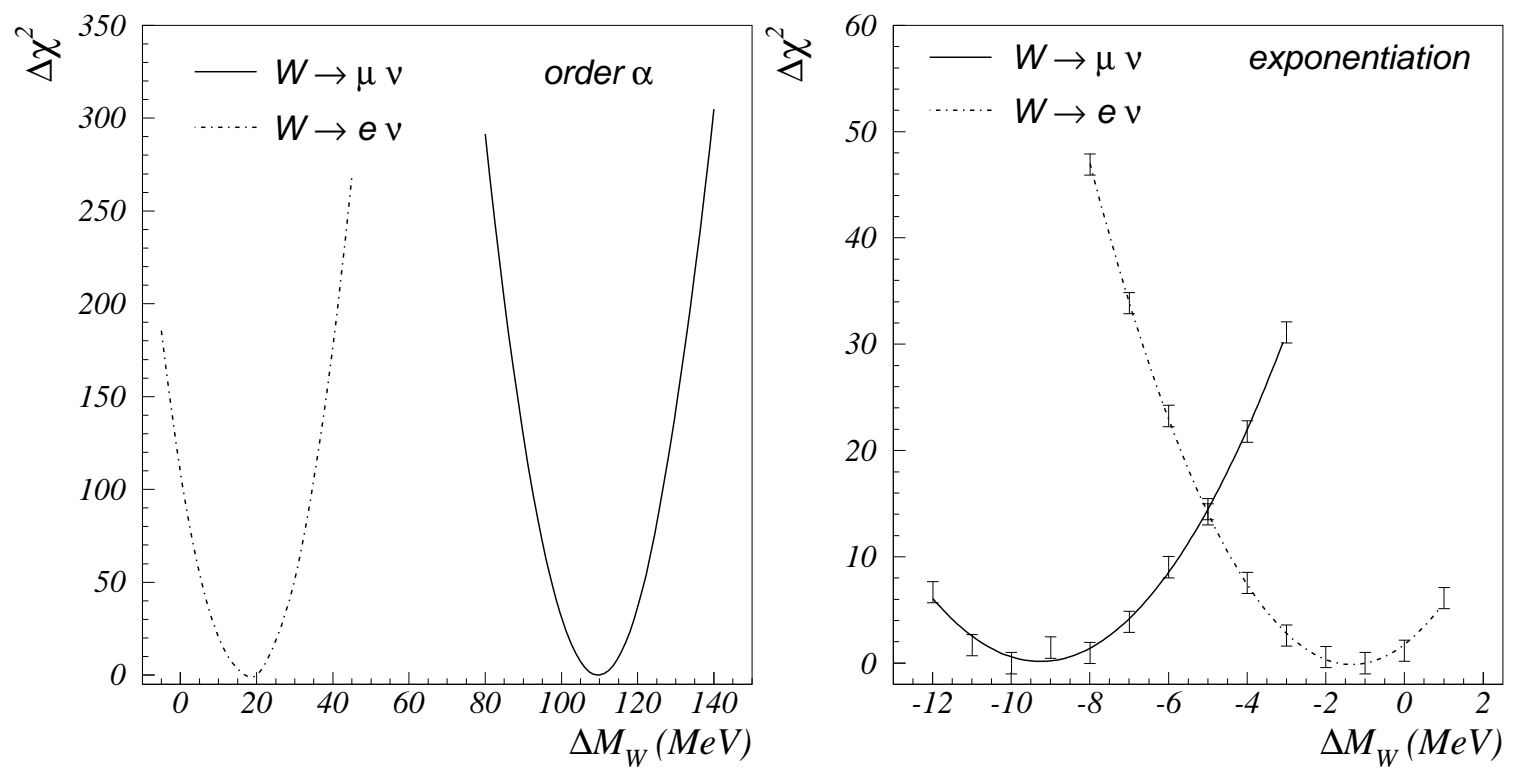

FIG. 2: The $\Delta \chi^{2}=\chi^{2}-\chi_{\min }^{2}$ distributions from a fit to the $M_{T}$ distribution, including $\mathcal{O}(\alpha)$ QED corrections (left) and higher-order QED corrections (right), as a function of the $W$ mass shift, at $\sqrt{s}=2 \mathrm{TeV}$. The results for the $W \rightarrow e \nu$ and $W \rightarrow \mu \nu$ channels are shown.

as a function of $\Delta M_{W} \equiv M_{W}-M_{W}^{r e f}$, for the fit with $\mathcal{O}(\alpha)$ corrections (left) and the fit with higher-order corrections (right). The mass shift observed for $\mathcal{O}(\alpha)$ corrections amounts to about $20 \mathrm{MeV}$ for the $W \rightarrow e \nu$ decay (dashed line) and to $110 \mathrm{MeV}$ for the $W \rightarrow \mu \nu$ decay (solid line), as a consequence of the different identification requirements. These shifts are in reasonable agreement with the results of the CDF and D $\varnothing$ collaborations, even in the absence of a complete detector simulation. The mass shift due to higher-order effects is about $10 \mathrm{MeV}$ for the $W \rightarrow \mu \nu$ channel (solid line) and a few $\mathrm{MeV}$ (dashed line) for the $W \rightarrow e \nu$ channel. We performed the same analysis for the LHC collider (using the cuts and pseudo-detector simulation of the Tevatron collider) and found that the same conclusions do apply to the LHC.

In conclusion, we have evaluated the impact of higher-order final-state QED corrections on the determination of the $W$ mass at hadron colliders, in view of future improved measurements with an accuracy of 15-30 MeV. In the presence of realistic selection criteria, we have found that the shift due to these corrections is about $10 \mathrm{MeV}$ in the $W \rightarrow \mu \nu$ channel and practically negligible in the $W \rightarrow e \nu$ channel. The calculation, if included in future experimental analyses, would reduce the uncertainty in the precision measurement of the $W$ mass at hadron colliders. To this end, the Monte Carlo program HORACE is available for data 
analysis. A more realistic analysis would require a full detector simulation, which is beyond the scope of the present paper. The study of the neutral-current process $p p(\vec{p}) \gamma, Z \rightarrow l^{+} l^{-}$ is left to a future work.

[1] U. Baur et al., in Proceedings of the Workshop on the Future of Particle Physics, Snowmass, July 2001, hep-ph/0111314.

[2] S. Haywood et al., in Proceedings of the Workshop on Standard Model Physics (and more) at the LHC, edited by G. Altarelli and M.L. Mangano, CERN Report 2000-04, p. 117; F. Gianotti and M. Pepe Altarelli, Nucl. Phys. Proc. Suppl. 89, 177 (2002).

[3] S. Catani et al., in Proceedings of the Workshop on Standard Model Physics (and more) at the LHC, edited by G. Altarelli and M.L. Mangano, CERN Report 2000-04, p. 1; U. Baur et al., in Proceedings of the Workshop QCD and Weak Boson Physics in Run II, edited by U. Baur, R.K. Ellis and D. Zeppenfeld, p. 115.

[4] D. Wackeroth and W. Hollik, Phys. Rev. D55, 6788 (1997).

[5] U. Baur, S. Keller and D. Wackeroth, Phys. Rev. D59, 013002 (1999).

[6] U. Baur, S. Keller and W.K. Sakumoto, Phys. Rev. D57, 199 (1998); U. Baur et al., Phys. Rev. D65, 033007 (2002).

[7] U. Baur and D. Wackeroth, hep-ph/0211089.

[8] U. Baur and T. Stelzer, Phys. Rev. D61, 073007 (2000).

[9] C.M. Carloni Calame et al., Nucl. Phys. B584, 459 (2000); C.M. Carloni Calame, Phys. Lett. B520, 16 (2001).

[10] W. Placzek and S. Jadach, hep-ph/0302065.

[11] T. Affolder et al., CDF Collaboration, Phys. Rev. D64, 052001 (2001).

[12] B. Abbott et al., DØ collaboration, Phys. Rev. D62, 092006 (2001).

[13] M. Caffo, R. Gatto and E. Remiddi, Nucl. Phys. B252, 378 (1985); M. Cacciari, G. Montagna and O. Nicrosini, Phys. Lett. B274, 473 (1992).

[14] WGRAD is available at the web site http://ubpheno.physics.buffalo.edu/ dow/wgrad.html.

[15] The Review of Particle Physics, K. Hagiwara et al., Phys. Rev. D66, 010001 (2002).

[16] J. Pumplin et al., JHEP 0207, 012 (2002).

[17] S. Abachi et al., DØ collaboration, Report FERMILAB-Pub-96/357-E. 